\title{
Continuous phase transition in a spin-glass model without time-reversal symmetry
}

\author{
G. Parisi* \\ Dipartimento di Fisica and INFN, Universita di Roma “La Sapienza," Piazzale Aldo Moro 2, 00185 Roma, Italy \\ M. Picco ${ }^{\dagger}$ \\ LPTHE, Université Pierre et Marie Curie, Paris VI and Université Denis Diderot, Paris VII, Boite 126, Tour 16, \\ $1^{\text {er }}$ étage, 4 place Jussieu, F-75252 Paris Cedex 05, France \\ and Departamento de Física, Universidade Federal do Espírito Santo, Vitória - ES, Brazil \\ F. Ritort ${ }^{\ddagger}$ \\ Departament de Física Fonamental, Facultat de Física, Universitat de Barcelona, Diagonal 647, 08028 Barcelona, Spain
}

(Received 15 December 1998)

\begin{abstract}
We investigate the phase transition in a strongly disordered short-range three-spin interaction model characterized by the absence of time-reversal symmetry in the Hamiltonian. In the mean-field limit the model is well described by the Adam-Gibbs-DiMarzio scenario for the glass transition; however, in the short-range case this picture turns out to be modified. The model presents a finite temperature continuous phase transition characterized by a divergent spin-glass susceptibility and a negative specific-heat exponent. We expect the nature of the transition in this three-spin model to be the same as the transition in the Edwards-Anderson model in a magnetic field, with the advantage that the strong crossover effects present in the latter case are absent. [S1063-651X(99)09806-2]
\end{abstract}

PACS number(s): 05.50.+q, 75.50.Lk, 64.60.Cn

\section{INTRODUCTION}

Nowadays there is large amount of research being done on the problem of the glass transition from the perspective of spin-glass theory $[1,2]$. This interest originates from old observations by Kirkpatrick, Thirumalai, and Wolynes [3], who found a striking similarity between the dynamical equations of some mean-field spin-glass models and the mode-coupling equations for glasses. The mode-coupling equations are characterized by the presence of a dynamical singularity at a temperature $T_{d}$ below which spin-spin correlation functions do not decay to zero in the infinite time limit signaling the breaking of ergodicity [4]. Above but close to $T_{d}$, the correlation functions display a plateau which separates two different relaxational regimes (the $\alpha$ and the $\beta$ processes). The family of models that show this behavior are those with one step of replica symmetry breaking (models with an infinite number of breaking steps describe better the spin-glass behavior found in strongly disordered magnets). These models are characterized by two singularities or transition temperatures. One transition is purely dynamical and corresponds to the mode-coupling transition $T_{d}$ previously described. The other transition at $T_{c}<T_{d}$ is thermodynamic and corresponds to a temperature below which replica symmetry breaks and the configurational entropy (also called complexity) vanishes. The transition at $T_{c}$ has features of both first- and second-order transitions like a discontinuity in the Edwards-Anderson order parameter and a finite jump in the specific heat.

\footnotetext{
*Electronic address: giorgio.parisi@roma1.infn.it

${ }^{\dagger}$ Electronic address: picco@1pthe.jussieu.fr

‡Electronic address: ritort@ffn.ub.es
}

It is widely accepted that the origin of the dynamical transition at $T_{d}$ (where the relaxation time diverges and ergodicity breaks) originates from the presence of an exponentially large number of states (exponentially large with the system size) which trap the system for exponentially large times forbidding the system to reach the equilibrium Gibbs measure. But it is also clear (and this was also recognized as a strong limitation in the original mode-coupling theory) that equilibrium below $T_{d}$ should be restored in finitedimensional systems where activated or nucleation process (i.e., jumps over finite free-energy barriers) takes place in a finite time. What is the final theory which correctly describes the nucleation processes taking place in glasses is one of the major open problems. The behavior of $T_{c}$ in the presence of short-range interactions is less clear. According to the meanfield picture, the transition at $T_{c}$ (where replica symmetry breaks) could well survive in finite dimensions. At $T_{c}$ the configurational entropy would still vanish. This scenario is merely (but now rephrased in the spin-glass language) the Adam-Gibbs-DiMarzio scenario (hereafter referred to as AGM) for the ideal glass transition $[5,6]$.

On top of this connection between the spin-glass theory and mode-coupling theory, a very interesting connection has also been established between the statics and dynamics of glassy systems in the off-equilibrium regime. In this case, the equilibrium order parameter for spin glasses [the so-called $P(q)$ function] is intimately related to the fluctuationdissipation ratio [2]. This link between statics and dynamics, originally suggested by the analysis of exactly solvable mean-field spin glasses $[7,8]$, is actually supported by extensive numerical simulations [9] and general arguments based on the assumption of linear-response theory applied to shortrange spin-glass models [10]. 
All these previous studies cannot predict how short-range corrections change the mean-field behavior. And in particular, it is unclear how the AGM scenario typical of a firstorder spin-glass transition is modified in finite dimensions. The answer may crucially depend on the presence of quenched disorder in the system and the mean-field critical behavior can be significantly altered in finite dimensions. Here, we will see that the mean-field scenario in the manner of AGM does not survive in finite dimensions for a certain class of models.

A preliminary account of some parts of this work has recently appeared in a different context [11] where a Binderlike parameter was proposed to study replica symmetrybreaking transitions. Also, different cases of the present model have already been studied in Refs. [12-14]. So our work complements these results, although the case we study here lacks time-reversal symmetry sharing some features of the Ising spin glass in a magnetic field.

The paper is divided as follows. In Sec. II we define the model, and the numerical algorithm is explained in Sec. III. In Sec. IV we present the equilibrium results obtained by simulating small systems. In Sec. V we present results for the order parameter and its cumulants. In Sec. VI we give results for a new parameter (to be defined later) which unambiguously shows the existence of a phase transition. Section VII is devoted to a discussion of the dynamical properties. Finally, in Sec. VIII, we summarize the main results and discuss some peculiar features of the present model.

\section{THE SHORT-RANGED $p$-SPIN MODEL}

The present model is a short-range generalization of the multispin interaction mean-field Ising spin glass defined by $[15,16]$

$$
\mathcal{H}=-\sum_{\left(i_{1}, i_{2}, \ldots, i_{p}\right)} J_{i_{1} i_{2}, \ldots, i_{p}} \sigma_{i_{1}} \sigma_{i_{2}}, \ldots, \sigma_{i_{p}}
$$

where the spins $\sigma_{i}, 1 \leqslant i \leqslant N$ ( $N$ is the size of the system) can take the values \pm 1 and the $J_{i_{1} i_{2}}, \ldots, i_{p}$ are quenched random variables with zero mean and variance $p ! /\left(2 N^{p-1}\right)$. In Eq. (1), all possible multiplets of $p$ spins interact through the random couplings $J_{i_{1} i_{2}}, \ldots, i_{p}$. Consequently there is no spatial dimensionality and the model retains its full meanfield character. In order to go beyond mean-field theory we need to suitably modify the model introducing short-ranged interactions in a finite-dimensional lattice. A possible way to modify Eq. (1) is to consider only links which couple nearest neighbors in a finite-dimensional regular lattice. For instance, we could locate the spins in the vertices of the lattice and consider only a certain set of triangles $(p=3)$, a certain set of squares $(p=4)$, and for a general $p$, only a certain set of plaquettes containing $p$ spins of the lattice $[17,18]$. In the presence of quenched disorder, such constructions have been considered in several cases [19-21]. In particular, in [21] a simple cubic lattice with $p=4$ was studied. Although freezing behavior was observed, no evidence of a finitetemperature transition was found in three dimensions. Unfortunately, this type of model with binary exchange couplings has a large ground-state degeneracy causing strong crossover effects (due to the presence of a nontrivial zero-temperature fixed point) at low temperatures. Furthermore, in this type of model the lower critical dimension seems to increase with $p$. This implies that large dimensions need to be studied in order to find a phase transition. More work is certainly necessary to identify whether the AGM scenario is valid in finite dimensions for this type of model.

The model we are going to study here is an alternative way to include short-range corrections. We locate $M$ different Ising spins $\sigma_{j}^{i_{1} i_{2}}, \ldots, i_{D}$ in each site of a regular cubic lattice. In this notation $j$ enumerates the different spins (it ranges from 1 to $M$ ) in a given site with coordinates $\left(i_{1}, i_{2}, \ldots, i_{D}\right)$, where $1 \leqslant i_{1}, i_{2}, \ldots, i_{D} \leqslant L$ and $L$ is the lattice size of the cubic lattice and $D$ is the dimensionality. The volume of the system is therefore given by $V=L^{D}$. The Hamiltonian is defined as follows:

$$
\mathcal{H}=\sum_{1 \leqslant i_{1}, i_{2}, \ldots, i_{p} \leqslant L} \sum_{\mu=1}^{D} \mathcal{H}_{\text {link }},
$$

where $\mathcal{H}_{\text {link }}$ is the Hamiltonian corresponding to the link defined by the site $\left(i_{1}, i_{2}, \ldots, i_{D}\right)$ and the direction $\mu, 1 \leqslant \mu$ $\leqslant D$. In our notations, a link is a pair $(P, \mu)$ which couples the point $P \equiv\left(i_{1}, i_{2}, \ldots, i_{D}\right)$ to the nearest-neighbor site in the $\mu$ direction $P+\mu \equiv\left(i_{1}, \ldots, i_{\mu}+1, \ldots, i_{D}\right)$. Note that in Eq. (2) each link is counted only once. For each link we sum all the possible groups of $p$ spins out of the $2 M$ spins located at nearest-neighboring sites of the lattice (with $p \leqslant 2 M$ ). The final expression for $\mathcal{H}_{\text {link }}$ is given by

$$
\begin{aligned}
\mathcal{H}_{\text {link }}= & -\sum_{k=1}^{p} \sum_{1 \leqslant a_{i} \leqslant M} \sum_{1 \leqslant b_{i} \leqslant M} \\
& \times J_{\text {link }}^{\left(a_{1}, \ldots, a_{k}, b_{1}, \ldots, b_{p-k}\right)} \\
& \times \sigma_{a_{1}}^{P} \sigma_{a_{2}}^{P} \cdots \sigma_{a_{k}}^{P} \sigma_{b_{1}}^{P+\mu} \sigma_{b_{2}}^{P+\mu} \cdots \sigma_{b_{p-k}}^{P+\mu} .
\end{aligned}
$$

The couplings $J_{\text {link }}^{\left(a_{1}, \ldots, a_{k}, b_{1}, \ldots, b_{p-k}\right)}$ are random variables (which take the values \pm 1 ) uncorrelated for different links $(P, \mu)$ and sets of $p$ spins $\left(a_{1}, \ldots, a_{k}, b_{1}, \ldots, b_{p-k}\right)$. Other versions of the model (for instance, $J_{\text {link }}^{\left(a_{1}, \ldots, a_{k}, b_{1}, \ldots, b_{p-k}\right)}=J^{\left(a_{1}, \ldots, a_{k}, b_{1}, \ldots, b_{p-k}\right)}$, i.e., translational invariant disorder) are also possible and they could have different properties.

As we observed in the Introduction, the present model has received considerable attention quite recently. A preliminary short account of our work was presented in [11]. In three dimensions, an exhaustive numerical study of the statics and the dynamics has been done in the case $M=4, p=4$ [12] while the case $M=3, p=4$ has been studied in [13]. So the main results on this model (except [11]) were obtained for the $p=4$ model. The study of the Gaussian propagators around the mean-field limit $M \rightarrow \infty$ as well as the $1 / M$ expansion were considered in [14]. The case we study here has the crucial property that the Hamiltonian (3) does not have the symmetry under time reversal (i.e., the global symmetry $\left.\sigma_{i} \rightarrow-\sigma_{i}, \forall i\right)$. 
Here we will present exhaustive results for the case $M$ $=2, p=3$ in $D=4$. We have chosen these parameters for the following reasons.

(i) $p=3$. This is the simplest case that lacks time-reversal symmetry. We expect in this case clearer results about the existence of replica symmetry-breaking transitions in shortrange systems.

(ii) $M=2$. This is the simplest nontrivial case. Larger values of $M$ require always more computational effort.

(iii) $D=4$. To be sure that we find a finite-temperature phase transition, we have studied a large dimensionality compatible with a reasonable computational effort.

For $p=3, M=2$ the general Hamiltonian Eq. (2) reads

$$
\begin{aligned}
\mathcal{H}= & -\sum_{i=1}^{V} \sum_{\mu=1}^{D}\left(J_{(11,10)}^{i, \mu} \sigma_{1}^{i} \sigma_{2}^{i} \sigma_{1}^{i+e_{\mu}}+J_{(11,01)}^{i, \mu} \sigma_{1}^{i} \sigma_{2}^{i} \sigma_{2}^{i+e_{\mu}}\right. \\
& +J_{(10,11)}^{i, \mu} \sigma_{1}^{i} \sigma_{1}^{i+e_{\mu}} \sigma_{2}^{i+e_{\mu}}+J_{(01,11)}^{i, \mu} \sigma_{2}^{i} \sigma_{1}^{i+e_{\mu}} \sigma_{2}^{\left.i+e_{\mu}\right)},
\end{aligned}
$$

where the $\left(e_{\mu} ; \mu=1, \ldots, D\right)$ denote the different unit vectors in a $D$-dimensional lattice. The $J$ 's are binary uncorrelated random variables and we will consider periodic boundary conditions.

Note that the model (4) has three spin interactions. One would simply expect the transition to belong to the class of $\phi^{3}$ theories. As we will see in the following sections, there is indeed a phase transition occurring in the present model. The absence of time-reversal symmetry in this model has crucial implications on the type of phase transition. We anticipate that the transition is related to the breaking of ergodicity at low temperatures, a consequence of the breaking of replica symmetry, the crucial symmetry to describe strongly disordered systems. We will try to clarify and give evidence on this point in forthcoming sections.

\section{THE NUMERICAL ALGORITHM}

We have studied the model in four dimensions using the parallel tempering method $[22,23]$. This is a good numerical method to equilibrate disordered systems at low temperatures. Contrary to the simulated tempering method [24,25], in this algorithm it is not necessary to determine the free energy at different temperatures to reach equiprobability in the occupancies of these temperatures. Although the parallel tempering is a very efficient method to surmount energy barriers, it is not clear how good the performance of the algorithm is in the presence of entropy barriers (i.e., when relaxation to equilibrium takes place along narrow channels or gutters in phase space).

The implementation of this algorithm is quite easy. It has been widely explained in the literature (for instance, see the reviews $[23,26,27]$ ) and we will limit ourselves to sketch the main steps of the algorithm. We consider a set of $N_{T}$ copies or replicas of the same system that stay at different temperatures $\left(T_{i} ; i=1, \ldots, N_{T}\right)$. Each copy or replica is then specified by a pair $(\mathcal{C}, i)$, where $\mathcal{C}$ denotes the microscopic configuration (i.e., the values of all the spins) and the temperature $T_{i}$ of the copy $i$. We can then construct a Markov process in the space of configurations plus temperatures which satisfies ergodicity and detailed balance by allowing the following moves. (i) Change configuration at fixed temperature: $(\mathcal{C}, i)$ $\rightarrow\left(\mathcal{C}^{\prime}, i\right)$ with probability

$$
P\left[(\mathcal{C}, i) \rightarrow\left(\mathcal{C}^{\prime}, i\right)\right]=\min \left(1, \exp \left\{-\beta\left[\mathcal{H}\left(\mathcal{C}^{\prime}\right)-\mathcal{H}(\mathcal{C})\right]\right\}\right)
$$

(ii) Exchange configurations of two systems at temperatures $\beta_{i}, \beta_{j}:\left\{(\mathcal{C}, i),\left(\mathcal{C}^{\prime}, j\right)\right\} \rightarrow\left\{\left(\mathcal{C}^{\prime}, i\right),(\mathcal{C}, j)\right\}$ with probability

$$
\begin{aligned}
P\left[\left\{(\mathcal{C}, i),\left(\mathcal{C}^{\prime}, j\right)\right\}\right. & \left.\rightarrow\left\{\left(\mathcal{C}^{\prime}, i\right),(\mathcal{C}, j)\right\}\right] \\
& =\min \left(1, \exp \left\{-\left(\beta_{i}-\beta_{j}\right)\left[\mathcal{H}(\mathcal{C})-\mathcal{H}\left(\mathcal{C}^{\prime}\right)\right]\right\}\right)
\end{aligned}
$$

The first type of move is the usual change of configuration at fixed temperature in the Monte Carlo method. The second move prevents the system from getting trapped in deep metastable minima. With this algorithm, configurations which are far from each other can be reached by allowing a single copy of the system to extract energy from other copies through a coupling mechanism step (2), Eq. (6) induced by the dynamics itself. Plainly speaking, the rest of the copies or replicas play the role of an external bath for a given copy. If a given copy remains trapped in a deep minima of the free energy, it can escape by extracting energy from the rest of the copies. The full Markov chain reaches thermal equilibrium when all temperatures are equally occupied and for each temperature the conditioned probability distribution $P(\mathcal{C} \mid i)$ is a Boltzmann distribution at temperature $1 / \beta_{i}$. In this way one simulates the model at different temperatures in the same run while being always in thermal equilibrium at different temperatures.

The time needed for the Markov process to reach the stationary distribution (i.e., the thermalization time) depends mainly on the choice of the set of temperatures and also on the ratio between the number of moves of the first (5) and second type (6). To solve the first problem, we have chosen a set of temperatures equally spaced in $\beta=1 / T$ in such a way that moves of the second type (6) do not occur with a too small probability at low temperatures. On the other hand, to uncorrelate the configurations as much as possible (in order to explore maximally distant configurations), it is convenient that the copies reach high enough temperatures. This is accomplished by enlarging the set of temperatures in the simulation up to twice the value of the critical temperature. For the present model we simulated 25 temperatures ranging from $T=2.0$ up to $T=5.0$ (as we will see, this corresponds to a window of temperatures covering the region $0.75 T_{c}$ $-2 T_{c}$ ). As previously remarked, a good thermalization can be achieved by choosing an appropriate number for the ratio between the number of tempering moves of the first and second type. If the number of moves of the second type is too small, then the system is not able to efficiently decorrelate and only a small number of excursions into the hightemperature region are performed. In the other extreme, the system decorrelates too fast and does not efficiently sample the landscape at a given temperature. We have tried several schemes intermediate between these two extremal cases and we have found that one move of the second type for each ten moves of the first type is a good compromise which efficiently thermalizes reasonable sizes. 


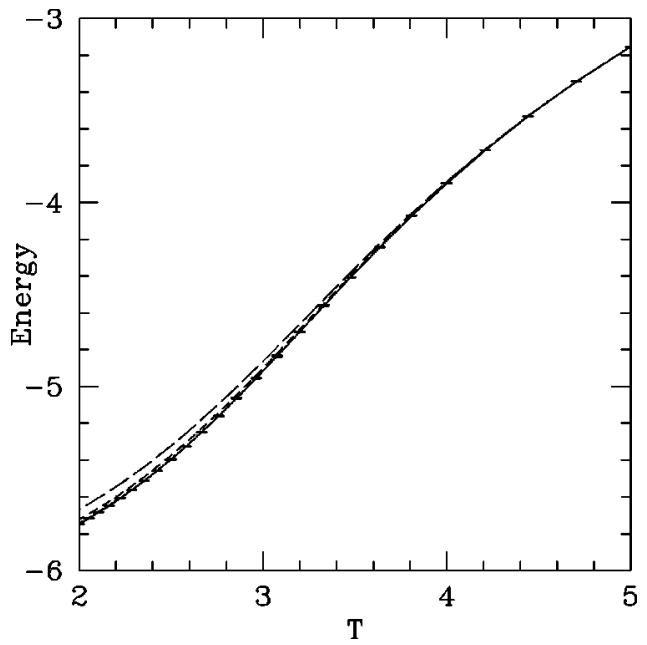

FIG. 1. Energy versus temperature ( $L=3,4,5,6$ correspond to long dashed, short dashed, dot, and solid lines). Error bars are shown for $L=6$.

We did extensive simulations for $L=3,4,5,6$ with $2^{15}$ $=32768 \mathrm{MCS}(L=3,4)$ and $2^{18}=262144(L=5,6) \mathrm{MCS}$ at each temperature. For sizes $L=3,4,5,6$ we studied 1000, 600, 300,100 samples, respectively. A preliminary study for a set of five samples showed that this number of steps was enough to reach thermal equilibrium for the selected sizes for the range of temperatures studied. This preliminary study turned out to be crucial to determine the smallest range of temperatures which allows the system to equilibrate. If the range of temperatures selected extends down to too low temperatures, then thermalization is hardly achieved. This means that the full Markov process associated to the parallel tempering algorithm does not reach the stationary Boltzmann solution. Then, there is no guarantee that thermalization is achieved either at low or at high temperatures.

\section{THERMODYNAMIC OBSERVABLES}

Preliminary research of the evidence for a phase transition includes the study of the temperature behavior of extensive quantities such as the internal energy or the specific heat. In Figs. 1 and 2 we show the internal energy and the specific heat averaged over the samples for different sizes as a function of temperature. The energy $E$ and the specific heat $C$ were computed using the expression

$$
\begin{gathered}
E=\overline{\langle\mathcal{H}\rangle}, \\
C=\frac{\beta^{2}}{N}\left(\overline{\left\langle\mathcal{H}^{2}\right\rangle}-\overline{\langle\mathcal{H}\rangle^{2}}\right) .
\end{gathered}
$$

In what follows, \langle\rangle stands for Gibbs average and $\overline{()}$ for disorder average. We note the absence of any jump in the internal energy as well as divergence or jump of the specific heat. This is a general result in phase transitions in strongly disordered systems and also applies in the present model. An important feature in Fig. 2 (also observed in other studies [12]) is the presence of a maximum in the specific heat at a temperature $(\simeq 3.2)$ much higher than $T_{c}$ (as we will see

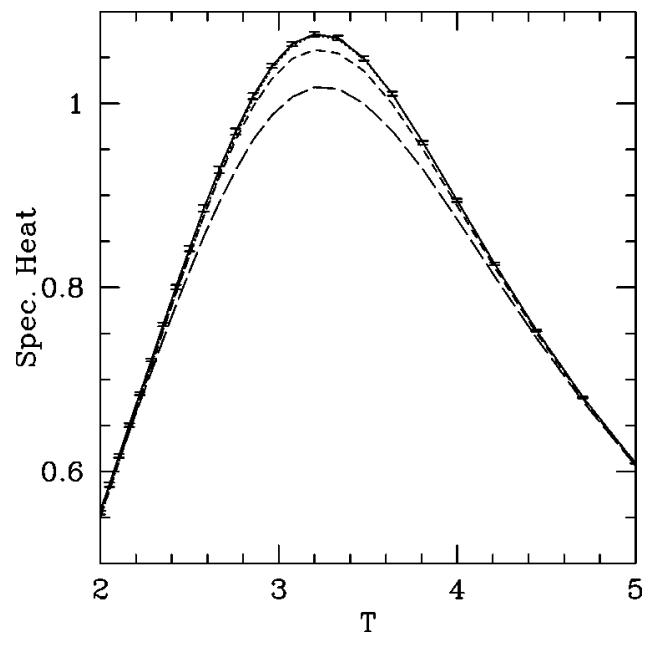

FIG. 2. Specific heat versus temperature ( $L=3,4,5,6$ correspond to long dashed, short dashed, dot, and solid lines). Error bars are shown for $L=6$.

later, $\left.T_{c} \simeq 2.62\right)$. At $T_{c}$ the specific heat is continuous so we expect the specific-heat exponent $\alpha$ to be negative.

\section{THE ORDER PARAMETER}

It was shown long ago by Edwards and Anderson that the appropriate order parameter for spin glasses is a measure of the temporal freezing of the local variables (in our case, the spins) of the system. In the general framework of spin-glass theory the order parameter is computed through the introduction of replicas in the system. This is the natural way of introducing the notion of a distance between two configurations in phase space. We take two replicas of the same system $\left\{\sigma_{i}, \tau_{i}\right\}$ [i.e., two identical Hamiltonians in Eq. (4) with identical realization of the couplings $J$ 's]. Then we define the global overlap $Q$ between the two replicas $N Q=\sum_{i=1}^{N} \sigma_{i} \tau_{i}$ and evaluate its probability distribution $P_{J}(q)$ averaged over the Gibbs measure $(\langle\rangle)$

$$
P_{J}(q)=\langle\delta(q-Q)\rangle
$$

In our specific model the overlap is $N Q=\Sigma_{i=1}^{N}\left(\sigma_{1}^{i} \tau_{1}^{i}\right.$ $+\sigma_{2}^{i} \tau_{2}^{i}$ ), where $\sigma_{1}^{i}, \sigma_{2}^{i}$ and $\tau_{1}^{i}, \tau_{2}^{i}$ occupy the site $i$ in the two different replicas, respectively. $P_{J}(q)$ gives the probability that two equilibrium configurations $\sigma_{i}, \tau_{i}$ have an overlap $q$. According to the mean-field scenario (the validity of which we would like to check for the present model), ergodicity breaks at low temperatures and the phase space splits up into a large number of single ergodic components or states. The barriers separating these components diverge with the size of the system suggesting that a symmetry is broken. This symmetry is generally referred to as replica symmetry and it is the symmetry under the group of permutations of a finite number of replicas. Somehow, this symmetry is artificial (actually, it emerges from the use of the replica trick, a general method to deal with the averaging of the logarithm of the partition function in disordered systems). But its physical meaning is quite appealing. Different equilibrium configurations $\sigma_{i}, \tau_{i}$ can take different values according to the basins of attractions (corresponding to different ergodic compo- 
nents) to which they belong. The function $P_{J}(q)$ is highly nontrivial and this is a signature that different configurations quite far from one another in the phase space contribute with a finite weight to the equilibrium partition function. Consequently, different states always have the same free energy, internal energy, and entropy per site but they differ only in the structure of their typical configurations. This is signaled by a nontrivial $P_{J}(q)$ distribution (i.e., with several peaks at different values of $q$ ).

Another general consequence of the splitting up of the phase space into different states is the possible existence of chaotic effects in the equilibrium phase [28,29]. A small perturbation in the Hamiltonian can change the shape of the states as well as reshuffle their Boltzmann weights. After perturbing the Hamiltonian, the new configurations can be very different from the initial ones. One consequence of this effect is the existence of non-self-averaging quantities. In particular, if we change completely the microscopic realization of the disorder in the original Hamiltonian [for instance, by changing the couplings $J$ in Eqs. (3) and (4) [31]], the new equilibrium states differ completely from the previous ones. In this case we do not add energy to the system (the new and the old states always have the same energy per site) but the reshuffling of the Boltzmann weights of the different states is enough to change completely the form of the $P_{J}(q)$. Then, the $P_{J}(q)$ is strongly non-self-averaging, a result which has been proved in mean-field theory and which we would like to check also in short-range systems.

The purpose of this section is to show how the study of the $P(q)=\overline{P_{J}(q)}$ averaged over the disorder can yield evidence for a phase transition in the present model. In the next section we will show that the non-self-averaging character of the $P_{J}(q)$ can be used as an independent check for the transition.

A good way to characterize the $P(q)$ is through its moments, in particular the first moment $\bar{q}$, the second cumulant which directly yields the spin-glass susceptibility $\chi_{\mathrm{SG}}$, the skewness $Y$, and the Binder parameter $Z$. More precisely, if we define the average $[f(q)]=\int d q f(q) P(q)$ [where $P(q)$ $\left.=\overline{P_{J}(q)}\right]$, then we have

$$
\begin{gathered}
\chi_{\mathrm{SG}}=V\left[(q-[q])^{2}\right], \\
Y=\frac{\left[(q-[q])^{3}\right]}{\left[(q-[q])^{2}\right]^{3 / 2}}, \\
Z=\frac{1}{2}\left(3-\frac{\left[(q-[q])^{4}\right]}{\left[(q-[q])^{2}\right]^{2}}\right) .
\end{gathered}
$$

In Figs. 3, 4, 6, and 7 we show $[q], \chi_{\mathrm{SG}}, Y$, and $Z$ as a function of temperature for different sizes. In Fig. 3 we show the first moment as a function of temperature. In the presence of time-reversal symmetry, $[q]$ vanishes (as well as the skewness $Y$ ) but not in the present case. Note that the curve of $[q]$ as a function of temperature is smooth without any sign of a jump or discontinuity. In mean-field spin-glass transitions (continuous or discontinuous), this result is expected because the jump in $[q]$ is proportional to $(1-m) q_{\mathrm{EA}}$, where $q_{\mathrm{EA}}$ is

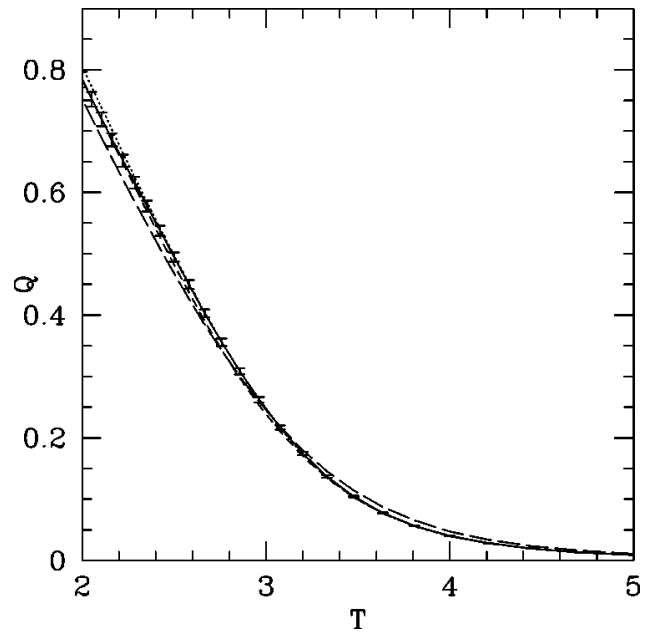

FIG. 3. First moment $[q]$ versus temperature ( $L=3,4,5,6$ correspond to long dashed, short dashed, dot, and solid lines). Error bars are shown for $L=6$.

the Edwards-Anderson parameter [the maximum value of $q$ such that $P(q) \neq 0$ ] and $m$ is the replica symmetry-breaking parameter (the size of the blocks in the breaking ansatz, see [30] for general introductory textbooks). In continuous replica symmetry-breaking transitions $q_{\mathrm{EA}}$ vanishes at $T_{c}$, but in first-order replica symmetry-breaking transitions $q_{\mathrm{EA}}$ is finite at $T_{c}$ and $m\left(T_{c}\right)=1$. In both cases there is no jump in $[q]$.

The results for the spin-glass susceptibility are more interesting. Experimentally, spin glasses show a divergence of the nonlinear susceptibility $\chi_{\mathrm{nl}}$ defined through the expansion

$$
M(H)=\chi_{0} H+\chi_{\mathrm{nl}} H^{3}+O\left(H^{5}\right) .
$$

It can be generally shown [30] that the nonlinear susceptibility is related to the spin-glass susceptibility defined in Eq. (10). Although the linear coefficient $\chi_{0}$ in the expansion (13) does not show any indication of $T_{c}$, the behavior of the nonlinear term $\chi_{\mathrm{nl}}$ is singular at $T_{c}$. In mean-field spin glasses with continuous transition, $\chi_{\mathrm{SG}}$ shows a power-law divergence at $T_{c}$. Contrarily, in mean-field spin glasses with

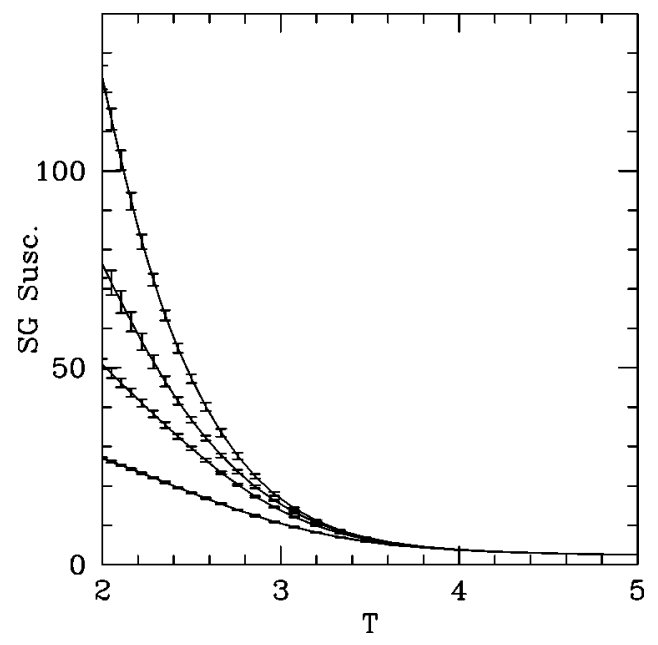

FIG. 4. $\chi_{\mathrm{SG}}$ versus temperature. From bottom to top, $L$ $=3,4,5,6$. 


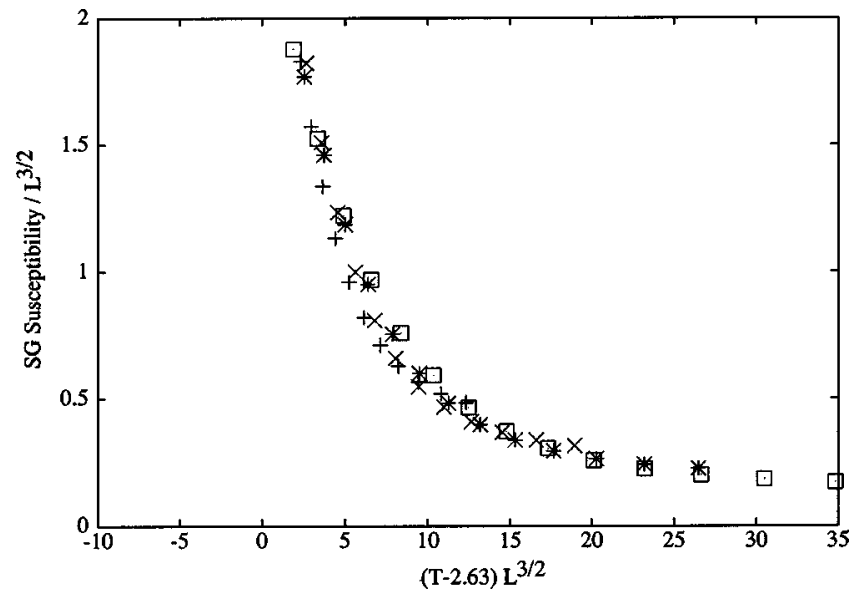

FIG. 5. Finite-size scaling of the spin-glass susceptibility. Our data are compatible with $\eta \simeq \frac{1}{2}, \nu=\frac{2}{3}$.

discontinuous RSB, $\chi_{\mathrm{SG}}$ shows a finite jump at $T_{c}$. This last feature has been claimed to be the explanation for the violation found in experiments at the glass transition for one of the two Ehrenfest relations [32]. Below $T_{c}, \chi_{\mathrm{SG}}$ is infinite in both cases. This result is related to the nontrivial character of the $P(q)$ which has contributions from different values of $q$. In Fig. 4 we show $\chi_{\mathrm{SG}}$ for different sizes as a function of $T$. Indeed, our results in Fig. 4 show an algebraic divergence of the spin-glass susceptibility $\chi_{\mathrm{SG}}$ and a least-squares fit of the data in the high-temperature region (where finite-size effects are negligible) yields $\chi_{\mathrm{SG}} \sim\left(T-T_{c}\right)^{-\gamma}$ with $T_{c} \simeq 2.63$ and $\gamma \simeq 1.0$. A finite-size scaling plot of the data for $\chi_{\mathrm{SG}} / L^{2-\eta}$ $\sim\left(T-T_{c}\right) L^{1 / \nu}$ is shown in Fig. 5 with $\nu \simeq \frac{2}{3}, \eta \simeq \frac{1}{2}$. This is in agreement with the exponents relation $\gamma=(2-\eta) \nu$ and with the previously estimated value of $\gamma$. Using the hyperscaling relation $\alpha=2-D \nu$ we get $\alpha \simeq-\frac{2}{3}$ in agreement with the absence of any singularity or jump in the specific heat (see Fig. 2). An independent measure of $\nu$ will be obtained in the following section. Anyway, from these first data, we may conclude that the divergence of $\chi_{\mathrm{SG}}$ is related to the divergence of a correlation length at $T_{c}$.

Figures 4 and 5 are the first evidence for a phase transition in the model. Figures 6 and 7 show the skewness and the

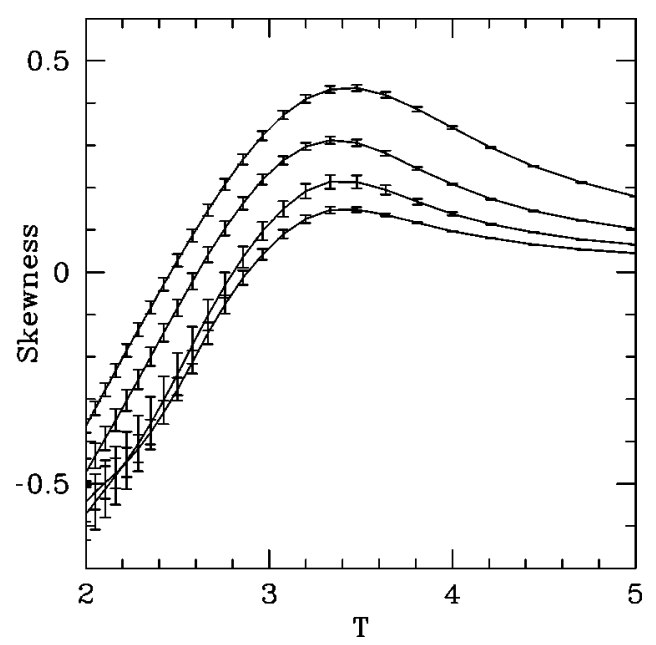

FIG. 6. Skewness versus temperature. From top to bottom, $L$ $=3,4,5,6$.

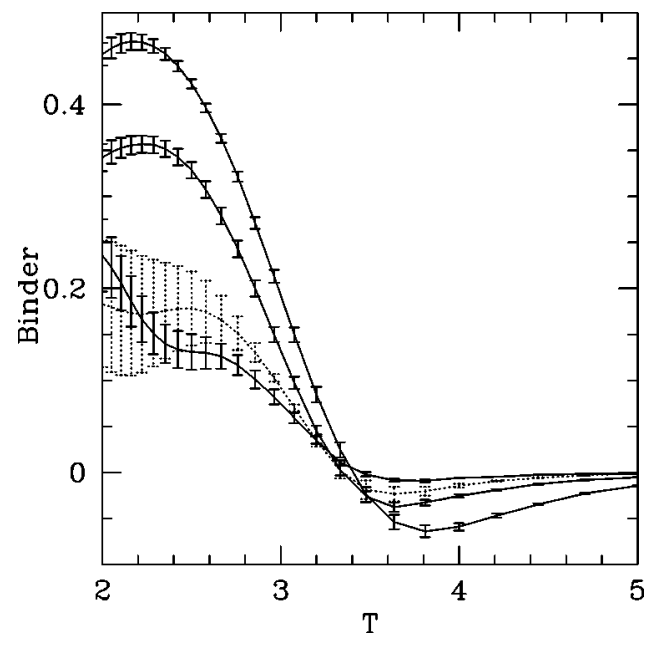

FIG. 7. Binder parameter versus temperature. From bottom to top in the high-temperature phase, $L=3,4,5,6$.

Binder parameter as a function of temperature. Because of the adimensional character of these quantities, one expects that they should be universal in the critical point and related to the amplitudes of the renormalization-group flow equations. In the large volume asymptotic regime the value of the skewness $Y$ and the Binder parameter $Z$ should be volume independent at $T_{c}$. Consequently, $T_{c}$ should manifest as a common crossing point of the curves corresponding to different system sizes. A common crossing point can hardly be identified in Figs. 6 and 7. Nevertheless, the fact that $Y$ and $Z$ do not vanish at low temperatures is a sign of a nontrivial low-temperature phase. Actually these two figures yield little information about the transition and it is hard to guess what is the character of the phase transition. Let us note that strikingly similar results were obtained for the Ising spin glass in a magnetic field [33].

Contrary to what is found in models with time-reversal symmetry, the $P(q)$ is not symmetric around $q=0$. This explains why $[q]$ and the skewness $(Y)$ are nonzero. Moreover, the fact that $Y<0$ is related to the asymmetric character of the $P(q)$. This is clearly shown in Figs. 8, 9, and 10 . Figure 8 shows the $P(q)$ for the largest simulated size $L$

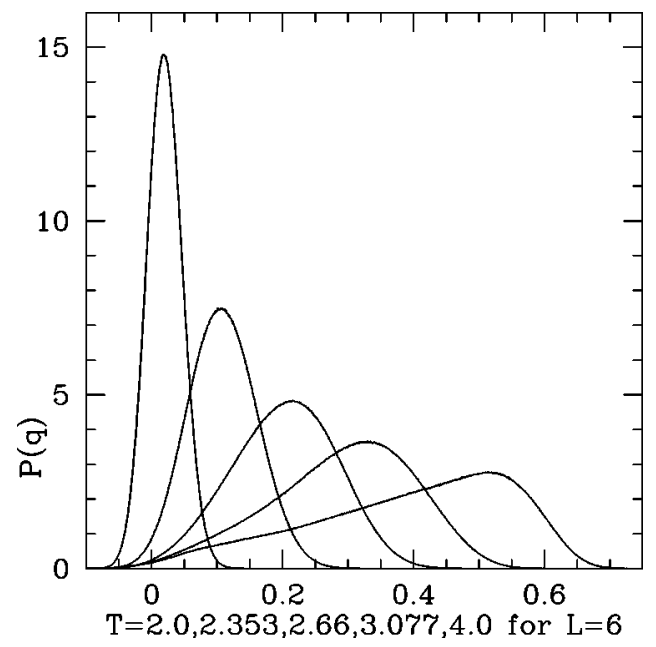

FIG. 8. $P(q)$ for $L=6$ at different temperatures (from left to right, $T=4.0,3.077,2.66,2.353,2.0)$. 


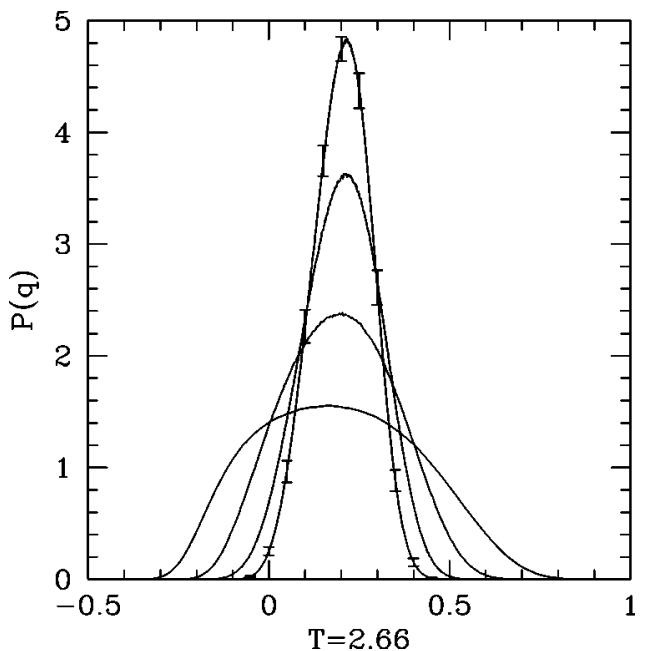

FIG. 9. $P(q)$ at $T \simeq T_{c}$ for $L=3,4,5,6$. For the sake of clarity, we show the error bars only for $L=6$ and only for some values of $q$.

$=6$ at different temperatures $T=4.0,3.077,2.66,2.353,2.0$. Note that above $T_{c}$ the $P(q)$ is Gaussian and develops a nontrivial shape at low temperatures with a nonvanishing tail which extends down to values of $q$ close to zero (the presence of this tail is clearly appreciated plotting the vertical axis in a logarithmic scale).

Except for the fact that the $P(q)$ is not symmetric around $q=0$, this behavior is reminiscent of what is observed in [12] for the $p=4$ case. Figures 9 and 10 show the $P(q)$ for different sizes at $T=2.66$ and at $T=2.0$, respectively.

\section{NEW EVIDENCE FOR THE TRANSITION}

New evidence for the existence of a phase transition can be obtained from the study of the sample-to-sample fluctuations of the order-parameter function $P_{J}(q)$. Our main observation originates from recent results obtained by Guerra [34]. Guerra has shown that sample-to-sample fluctuations of the cumulants of the order-parameter distribution $P_{J}(q)$ are Gaussian distributed in the thermodynamic limit. Let us now

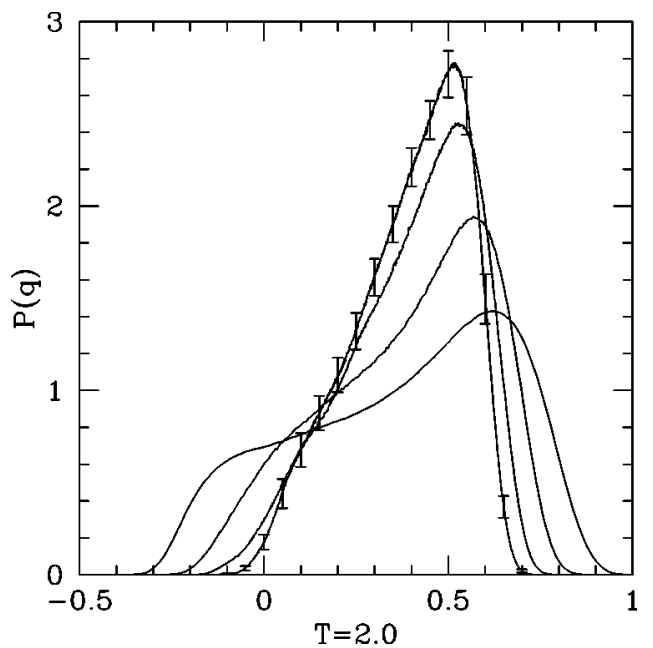

FIG. 10. $P(q)$ at $T=2.0$ for $L=3,4,5,6$. Errors are shown for $L=6$, for some values of $q$.

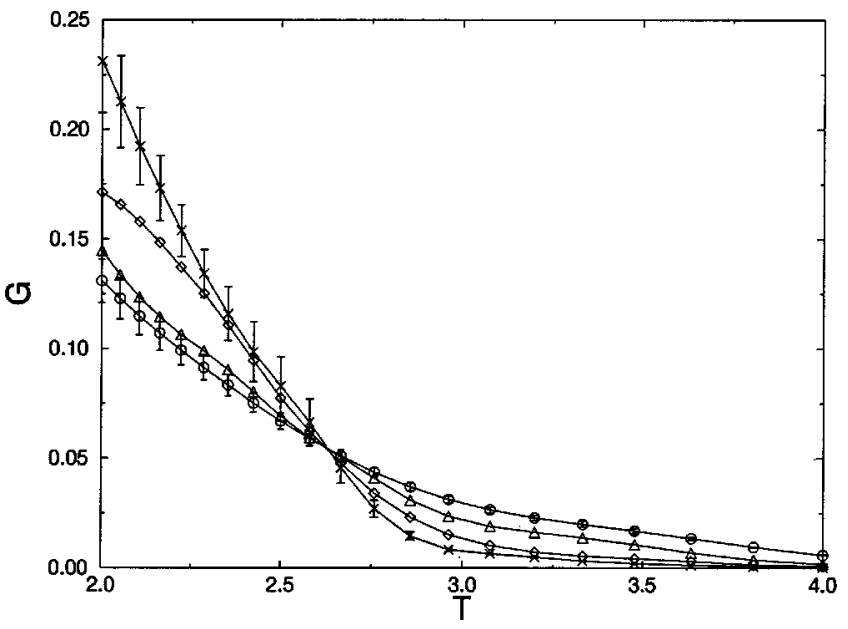

FIG. 11. Parameter $G$ for $L=3,4,5,6$ (open circles, triangles, diamonds, and crosses, respectively).

define a sample-dependent (i.e., $J$ dependent) susceptibility through

$$
\chi_{\mathrm{SG}}^{J}=V\left(\left\langle q^{2}\right\rangle-\langle q\rangle^{2}\right) .
$$

Note that $\overline{\chi_{\mathrm{SG}}^{J}}$ is different from $\chi_{\mathrm{SG}}$ defined in Eq. (10). It can be proved [34] that the following relationship is fulfilled in spin glasses below $T_{c}$ :

$$
G=\frac{\overline{\left(\chi_{\mathrm{SG}}^{J}\right)^{2}}-\left(\overline{\chi_{\mathrm{SG}}^{J}}\right)^{2}}{\overline{V^{2}\left\langle(q-\langle q\rangle)^{4}\right\rangle}-\left(\overline{\chi_{\mathrm{SG}}^{J}}\right)^{2}}=\frac{1}{3},
$$

where, as before, $\overline{()}$ means average over the quenched disorder.

The interest of defining the parameter $G$ is that it vanishes above the transition temperature in the disordered phase where sample-to-sample fluctuations of the $P_{J}(q)$ disappear in the $V \rightarrow \infty$ limit. Similar information to that obtained from Eq. (15) can also be gathered from the sample-to-sample fluctuations of $\chi_{\mathrm{SG}}^{J}$,

$$
A=\frac{\overline{\left(\chi_{\mathrm{SG}}^{J}\right)^{2}}-\left(\overline{\chi_{\mathrm{SG}}^{J}}\right)^{2}}{\left(\overline{\chi_{\mathrm{SG}}^{J}}\right)^{2}} .
$$

In principle, Eq. (16) also yields nontrivial behavior in the low-temperature phase even though (in contrast to $G$ ) it does not necessarily converge (in the thermodynamic limit) to a temperature-independent value. Both parameters $(A$ and $G)$ are good indicators of the transition although only $A$ gives a precise answer to the question of whether the order parameter is self-averaging or not. The reason [35] is that $G$ may be finite even when the numerator and denominator in Eq. (15) vanish. Actually $A$ is the numerator of $G$ so it gives precise information as to whether self-averaging is satisfied [36].

Note that $G$ is a parameter which plays the same role as the usual Binder parameter $g$ in ferromagnets and is given (in the $V \rightarrow \infty$ limit) by $G(T)=(1 / 3)\left[1-\Theta_{H}\left(T-T_{c}\right)\right]$, where $\Theta_{H}$ is the Heaviside theta function. In RSB transitions, Eq. 


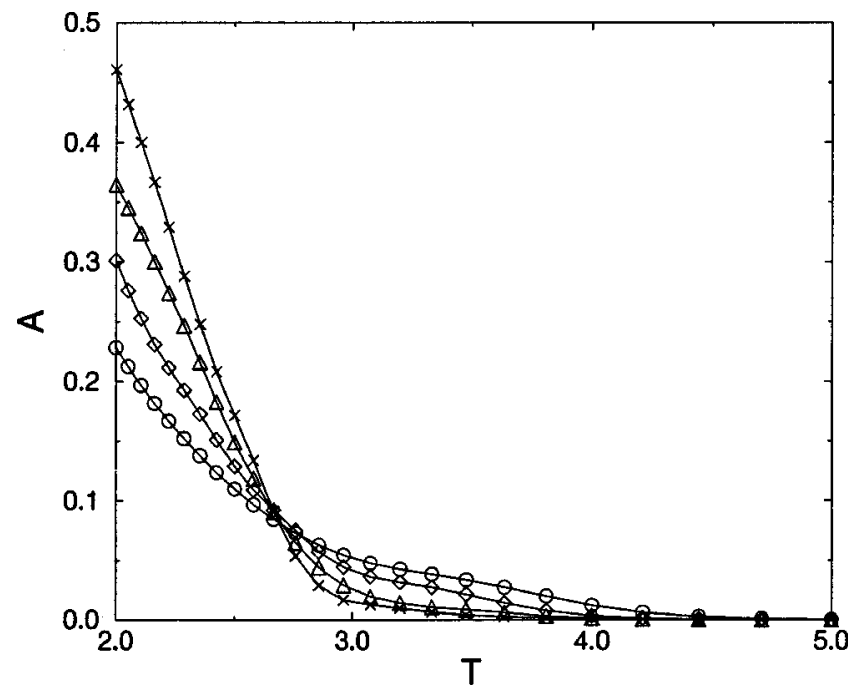

FIG. 12. Parameter $A$ for $L=3,4,5,6$ (open circles, rhombi, triangles, and crosses, respectively).

(15) goes to zero (as the size $V$ increases) as $1 / V$ for $T>T_{c}$ but converges to a finite value for $T<T_{c}$. We expect the critical temperature (where RS breaks) to be signaled by the crossing of the different curves corresponding to different lattice sizes.

Our results for $G$ and $A$ are shown in Figs. 11 and 12 . Both figures show essentially the same result, i.e., the curves for $G$ and $A$ for different sizes display a common crossing point located approximately at $T_{c} \simeq 2.63$ in agreement with the result derived in the preceding section from the divergence of the spin-glass susceptibility. Assuming for the parameter $G$ the following scaling behavior $G(T)=\hat{G}(L / \xi)$ with $\xi \sim\left(T-T_{c}\right)^{-\nu}$, then $(d G / d T)_{T=T_{c}} \sim L^{1 / \nu}$. In Fig. 13 we show the scaling behavior for $A$ and $G$. The scaling plots for $A$ and $G$ yield a more precise fit to the critical exponent $\nu$ because it involves only one free parameter $\left(T_{c}\right.$ was obtained looking at the crossing of the different curves). A good estimate for $\nu$ yields $\nu \simeq \frac{2}{3}$ for both $A$ and $G$ but precision is not good enough to exclude a slightly smaller value (such as $\nu$ $=\frac{1}{2}$ ). The value $\frac{2}{3}$ is in good agreement with the one obtained from the divergence of the spin-glass susceptibility.

Figures 11 and 12 clearly suggest that replica symmetry

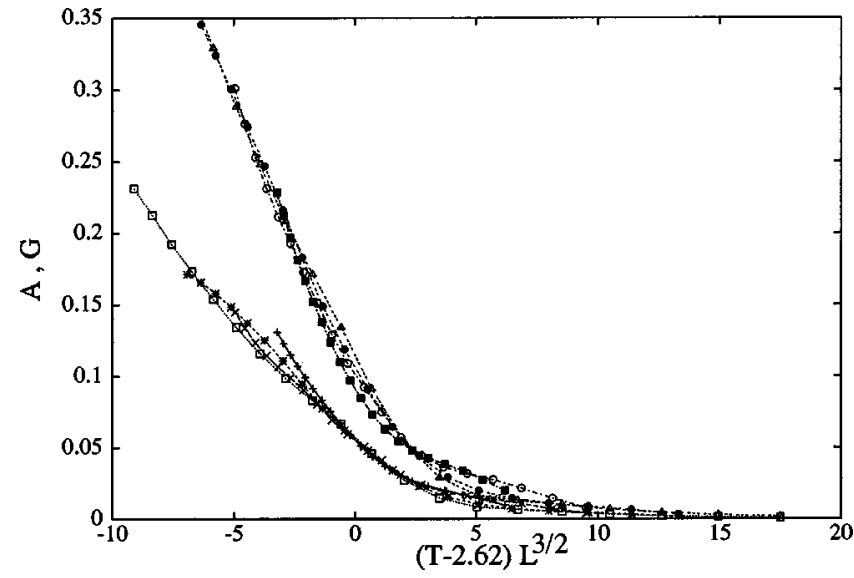

FIG. 13. Parameters $A, G$ for different sizes $L=3,4,5,6$ versus $(T-2.62) L^{3 / 2}$.

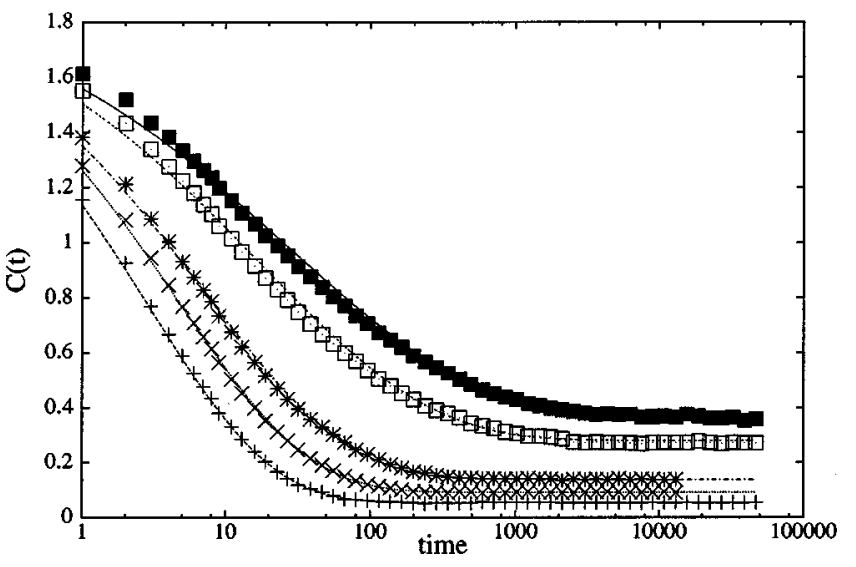

FIG. 14. $C(t)$ at $\beta=0.26(+), \beta=0.28(\times), \beta=0.30(*), \beta$ $=0.34(\square), \beta=0.36(\square)$, for one sample with $L=20$.

breaking takes place below $T_{c}$. This supports the result that the breaking of ergodicity is intimately related to the nonself-averaging character of the order parameter. This result is in contradiction with heuristic arguments by Newman and Stein [37], who have suggested that self-averaging should be automatically restored in short-range systems due to the translational invariance symmetry of the lattice.

\section{THE DYNAMICAL EXPONENT $Z$}

Now that we have corroborated the existence of a thermodynamic phase transition in the model, we would like to learn more about its nature. In particular, we would like to clarify whether the relaxation time (which is analogous to the shear viscosity of real glasses) shows an anomalous behavior in the vicinity of the glass region. It is well known that an activated behavior in the relaxation time is one of the main characteristics in real glasses. On the other hand, a powerlaw divergence of the relaxation time is a signature for a second-order phase transition where a massive mode vanishes.

We have computed the equilibrium time correlation function $C(t)$ at several temperatures above the estimated $T_{c}$. $C(t)$ is defined through

$$
C(t)=\frac{1}{V} \sum_{i=1}^{V}\left[\sigma_{1}^{i}(0) \sigma_{1}^{i}(t)+\sigma_{2}^{i}(0) \sigma_{2}^{i}(t)\right] .
$$

Compared to similar studies undertaken in the $p=4$ case $[12,13]$, the analysis in the present case turns out to be more difficult. This is due to the fact that in the absence of timereversal symmetry in the Hamiltonian, $C(t)$ does not decay to zero and there is one more unknown parameter $[C(\infty)]$. To have a reasonable estimate of $z$, we did two types of measures. On the one hand we measured $C(t)$ and fitted it to a stretched exponential form

$$
C(t)=q+(2-q) \exp \left(-(t / \tau)^{\beta}\right)
$$

with three free parameters $q[C(\infty)], \tau$ (the relaxation time), and $\beta$ (the stretching exponent). Note that $C(t)$ is normalized such that $C(0)=2$. Figure 14 shows some of the fits which turn out to be quite good. In this way we got some estimates for $\tau$ which unfortunately are not very precise, to 


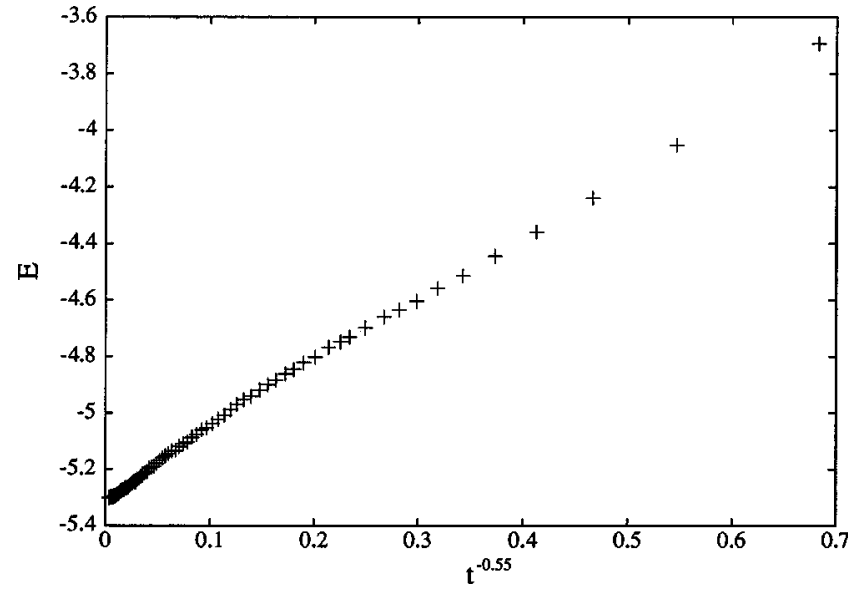

FIG. 15. Energy versus $t^{-0.55}$ at $T=2.62$ for one sample with $L=20$.

determine the value of $z$. To be more precise, one should include a power-law term $t^{-\alpha}$ multiplying the exponential term in the fitting function (18) as was done by Ogielsky in the study of the three-dimensional Edwards-Anderson model [38]. Unfortunately, including a term of this type in Eq. (18) introduces too many free parameters into $C(t)$, making fits poorly predictive. Nevertheless, from Fig. 14, we may conclude that relaxation turns out to be very slow close to $T_{c}$.

Our values estimated for the relaxation time exclude any activated behavior. This excludes the existence of a viscosity anomaly as well as the existence of two step relaxation processes in this model. The same conclusion was reached in the $p=4, D=3$ case by studying the $C(t)$ [12].

An estimate for $z$ can be obtained by studying the offequilibrium decay of the order parameter [39] or the internal energy [40]. This last case has been applied also to the study of structural glass models [41] as well as in the $p=4$ case [12]. In this case, one studies the decay of the internal energy starting from a random initial configuration at $T=T_{c}$ and using a fit to a power-law behavior of the following form:

$$
E(t)=E(\infty)+A t^{-\lambda} \text {. }
$$

Note that this is an off-equilibrium measure which is expected to yield the equilibrium dynamical exponent. Under the assumption that hyperscaling is valid, and using simple scaling relations, one obtains the exponents relation $\lambda=(d$ $-1 / \nu) / z$. In Fig. 15 we show the decay of the internal energy at the estimated $T_{c}$. A good fit is obtained with $\lambda=0.55$ \pm 0.1 , which yields $z \simeq 4.5 \pm 1$. This value for $z$ is very similar to the one found in the Edwards-Anderson model in four dimensions. Still, in this model the divergence of the relaxation time is not fast $(z \nu \simeq 3)$ if compared with that of the $M=3, p=4$ model $[12,13](z \simeq 7, z \nu \simeq 6 \pm 1)$. The value of $z \nu$ being not very large in our model (at least compared to those generally found in spin-glass models in three dimensions) explains why we succeeded in getting very clean results through finite-size scaling for the existence of a phase transition. Thermalization was easier to achieve.

\section{DISCUSSION}

In this paper we have investigated the critical behavior of a three-spin model in finite dimensions. The motivation was to understand how short-range effects modify the AGM mean-field scenario for the spin-glass transition. Moreover, the Hamiltonian for the present model $(M=2, p=3)$ has no time-reversal symmetry. In the presence of a thermodynamic transition, this lack of time-reversal symmetry has far reaching consequences on the nature of the transition.

We have established (through finite-size scaling methods) the existence of a phase transition without latent heat and with an algebraic divergence of the spin-glass susceptibility. On the other hand, we find indications that the relaxation time diverges according to an algebraic power law as in ordinary continuous phase transitions excluding the presence of an activated relaxation time.

Consequently, we are led to the conclusion that the firstorder character of the transition present in the mean-field limit $(M \rightarrow \infty)$ is lost in finite dimensions. In particular, our results $\nu \simeq \frac{2}{3}, \eta \simeq \frac{1}{2}, \gamma \simeq 1, z \simeq 4.5$ yield reasonable fits to all the data. Moreover, these exponents yield a negative value for the specific-heat exponent in agreement with the fact that there is not jump or divergence in the specific heat at $T_{c}$. Note that $\alpha$ vanishes in mean field so the main effect of finite-dimensional corrections is to decrease the value of $\alpha$. A negative value for $\alpha$ was apparently also obtained for $p$ $=4$ in three dimensions for $M=3,4$. Although large size simulations in $p=4, D=3$ [13] yield a smaller value of $\nu$ (compatible with $\nu=2 / D$ and hence $\alpha=0$ ), we must exclude this possibility from the absence of any jump in the specific heat at $T_{c}$.

Quite long ago, Gross, Kanter, and Sompolinsky, from the solution of the mean-field Potts glass [42], suggested the possibility that $\nu=2 / D$ could be valid in finite dimensions similar to what happens for pure systems (with the corresponding relation $\nu=1 / D)$. In particular, the explanation for the rounding of the phase transition due to finite-size effects would be very similar to the explanation valid in first-order transitions in pure systems $[43,44]$ but now modified to account for the presence of randomness. If the transition in finite dimensions were first order, then we would expect the validity of the relation $\nu=2 / D$ as well as the absence of upper critical dimension. Our numerical results tend to discard this possibility.

Note that the model we are considering here has no timereversal symmetry. Consequently, any thermodynamic transition cannot be associated with the breaking of an original symmetry of the Hamiltonian. In this respect, the transition we are facing closely resembles the mean-field transition of spin glasses in a magnetic field. We believe that the type of transition presented here is one of the most clear examples of second-order phase transition in strongly disordered systems where replica symmetry breaks. Figures 11, 12, and 13 offer good evidence for this result.

Finally, we would like to comment on the behavior of the entropy of the model as a function of the temperature. One prominent prediction in the AGM scenario is the collapse of the configurational entropy at $T_{c}$. Obviously, in the present model we do not expect that the configurational entropy vanishes at $T_{c}$ since the transition is continuous. In Fig. 16 we plot the total entropy (which are the sum of the configurational and its intrastate part) as a function of $\beta$ obtained numerically by integrating the internal energy between $\beta$ $=0$ and $\beta$. As $\beta$ increases (data are shown between $\beta=0$ 


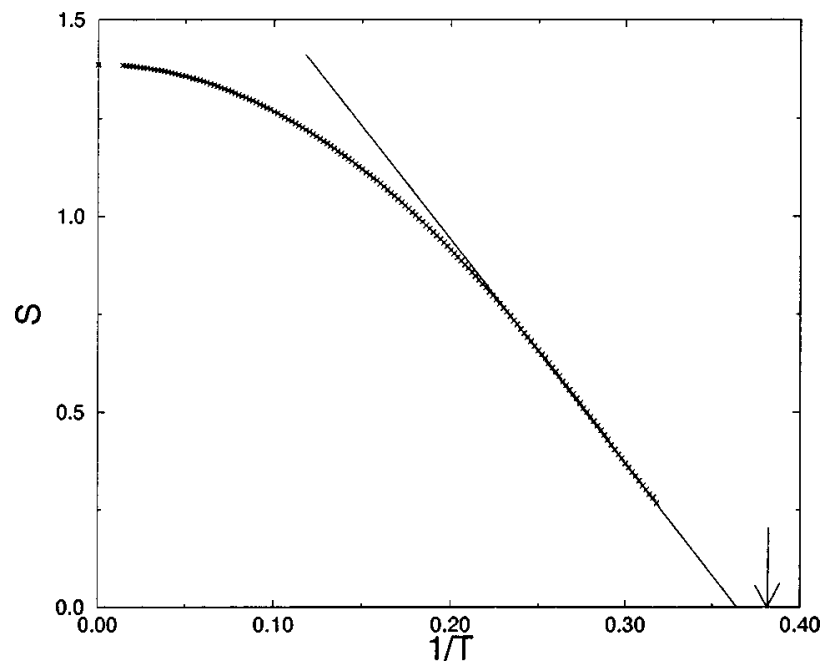

FIG. 16. Entropy of the model versus temperature. The continuous line is a linear extrapolation of the last set of points. Data were obtained simulating a sample with $L=10$. The arrow corresponds to the estimated value of $T_{c}$.

and $\beta=0.32$ ), the entropy becomes steadily linear and seems to extrapolate to zero at a finite value. A linear fit to the last set of data yields $S(\beta) \simeq 5.78(0.364-\beta)$, which vanishes at $T_{c} \simeq 2.75$, a result strikingly close to the previously estimated value of $T_{c}$ through finite-size scaling methods. This value of $T_{c}$ is quite stable against data inclusion or exclusion in the fit. Actually the same linear behavior is observed for the entropy when plotted versus the temperature. A linear fit yields in that case $S(T) \simeq 0.536(T-2.64)$, a result still very close to previous estimates of $T_{c}$. Consequently, the total entropy (and also the configurational entropy) seems indeed to vanish at a temperature slightly above $T_{c}$. This is nonsense because above $T_{c}$ the entropy must always be finite. We conclude that the entropy must stop decreasing at tem- peratures close to $T_{c}$ and depart from the linear behavior again. Actually, this is what we expect from the presence of the maximum of the specific heat (Fig. 2). Using the relation $C=T(\partial S / \partial T)$, we safely predict a breakdown of the linear behavior at $T \simeq 3.2(\beta \simeq 0.31)$ where the entropy should start to form a plateau.

To conclude, we have found that short-range effects in some class of models (with nontranslational invariant disorder) cause the transition to become second order. The type of transition should be the same as the one expected for the Edwards-Anderson model in a magnetic field. The main difference is that, in the last case, the parameter space contains the temperature and the field while in the former case the only parameter which controls the phase transition is the temperature. This implies strong crossover effects in the critical region for the Edwards-Anderson model in a field due to the proximity of the zero-field fixed point. Such crossover effects are not present in the present model, making the determination of the critical behavior much simpler. It would be very interesting to extend the research of strongly disordered spin models without time-reversal symmetry to other cases, such as models with translationally spatial invariant disorder, to understand under which conditions the first-order character of the mean-field transition survives in finite dimensions.

\section{ACKNOWLEDGMENTS}

We acknowledge Matteo Campellone for discussions and a careful reading of the manuscript. F.R. acknowledges FOM and the University of Amsterdam for financial support, where part of this work was done, and Ministerio de Educacion y Ciencia in Spain for support through project PB970971. M.P. acknowledges CAPES (Brazil) for financial support and the Physics Department of the UFES, and in particular J. Fabris, for hospitality.
[1] Proceedings of the XIV Sitges Conference, Complex Behavior of Glassy Systems, edited by M. Rubi and C. Perez-Vicente (Springer-Verlag, Berlin, 1997).

[2] J. P. Bouchaud, L. F. Cugliandolo, J. Kurchan, and M. Mezard, in Spin Glasses and Random Fields, edited by A. P. Young (World Scientific, Singapore, 1997)

[3] T. R. Kirkpatrick and D. Thirumalai, Phys. Rev. B 36, 5388 (1987); T. R. Kirkpatrick and P. G. Wolynes, ibid. 36, 8552 (1987). A review of the results of these authors and further references can be found in T. R. Kirkpatrick and D. Thirumalai, Transp. Theory Stat. Phys. 24, 927 (1995).

[4] W. Götze, Liquid, Freezing and the Glass Transition, Les Houches, edited by J. P. Hansen, D. Levesque, and J. ZinnJustin (North-Holland, Amsterdam, 1989), p. 287.

[5] J. H. Gibbs and E. A. Di Marzio, J. Chem. Phys. 28, 373 (1958).

[6] G. Adams and J. H. Gibbs, J. Chem. Phys. 43, 139 (1965).

[7] L. F. Cugliandolo and J. Kurchan, Phys. Rev. Lett. 71, 173 (1993).

[8] S. Franz and M. Mézard, Europhys. Lett. 26, 209 (1994).
[9] E. Marinari, G. Parisi, F. Ricci-Tersenghi, and J. J. RuizLorenzo, J. Phys. A 31, 2611 (1998).

[10] S. Franz, M. Mezard, G. Parisi, and L. Peliti, e-print cond-mat/9803108 [Phys. Rev. Lett. (to be published)].

[11] E. Marinari, C. Naitza, G. Parisi, M. Picco, F. Ritort, and F. Zuliani, Phys. Rev. Lett. 81, 1698 (1998).

[12] M. Campellone, B. Coluzzi, and G. Parisi, e-print cond-mat/9804291 [Phys. Rev. B (to be published)].

[13] S. Franz and G. Parisi, e-print cond-mat/9805088.

[14] M. Campellone, G. Parisi, and P. Ranieri, e-print cond-mat/9806235 [Phys. Rev. B. (to be published)].

[15] D. J. Gross and M. Mezard, Nucl. Phys. B 240, 431 (1984).

[16] E. Gardner, Nucl. Phys. B 257, 747 (1985).

[17] O. G. Mouritsen, B. Frank, and D. Mukamel, Phys. Rev. B 27, 3018 (1983).

[18] A. Cappi, P. Colangelo, G. Gonnella, and A. Maritan, Nucl. Phys. B 370, 659 (1992).

[19] H. Rieger, Physica A 184, 279 (1992).

[20] J. Kisker, H. Rieger, and H. Schreckenberg, J. Phys. A 27, L853 (1994). 
[21] D. Alvarez, S. Franz, and F. Ritort, Phys. Rev. B 54, 9756 (1996).

[22] K. Hukushima and K. Nemoto, J. Phys. Soc. Jpn. 65, 1604 (1996).

[23] E. Marinari, G. Parisi, and J. J. Ruiz-Lorenzo, in Spin Glasses and Random Fields, edited by A. P. Young (World Scientific, Singapore, 1997).

[24] B. A. Berg and T. Celik, Phys. Rev. Lett. 69, 2292 (1992).

[25] E. Marinari and G. Parisi, Europhys. Lett. 19, 451 (1992).

[26] B. A. Berg, e-print hep-lat/9705011, and references therein.

[27] E. Marinari, e-print cond-mat/9612010.

[28] I. Kondor, J. Phys. A 22, L163 (1989).

[29] F. Ritort, Phys. Rev. B 50, 6844 (1994).

[30] M. Mézard, G. Parisi, and M. A. Virasoro, Spin Glass Theory and Beyond (World Scientific, Singapore, 1987); K. Binder and A. P. Young, Rev. Mod. Phys. 58, 801 (1986); K. H. Fischer and J. A. Hertz Spin Glasses (Cambridge University Press, Cambridge, 1991).

[31] V. Azcoiti, E. Follana, and F. Ritort, J. Phys. A 28, 3863 (1995).
[32] T. M. Nieuwenhuizen, Phys. Rev. Lett. 79, 1317 (1997).

[33] M. Picco and F. Ritort, Physica A 250, 46 (1998).

[34] F. Guerra, Int. J. Mod. Phys. B 10, 1675 (1996).

[35] H. Bokil, A. J. Bray, B. Drossel, and M. A. Moore, Comment to [11], e-print cond-mat/9811304.

[36] E. Marinari, C. Naitza, G. Parisi, M. Picco, F. Ritort, and F. Zuliani, Response to preceding comment, e-print cond-mat/9811080.

[37] C. M. Newman and D. L. Stein, Phys. Rev. E 55, 5194 (1997).

[38] A. T. Ogielsky, Phys. Rev. B 34, 6586 (1986).

[39] R. E. Blundell, K. Humayun, and A. J. Bray, J. Phys. A 25, L733 (1992).

[40] B. Coluzzi and G. Parisi, e-print cond-mat/9712261 [J. Phys. A (to be published)].

[41] G. Parisi, e-print cond-mat/9801034.

[42] D. J. Gross, I. Kanter, and H. Sompolinsky, Phys. Rev. Lett. 55, 304 (1985).

[43] B. Nienhuis and M. Nauenberg, Phys. Rev. Lett. 28, 240 (1972).

[44] M. E. Fisher and A. N. Berker, Phys. Rev. B 26, 2507 (1982). 